\title{
Research on Key Technical Indexes of Coastal Reclamation Planning and Design
}

\author{
Zhang Shixia, ${ }^{1, *}$, Zhan Zhenyu $^{2}$, and Cao Guangtian ${ }^{1}$ \\ ${ }^{1}$ Zhejiang Tongji Vocational College of Science and Technology, Hangzhou 311231, China \\ ${ }^{2}$ Huadong Engineering Corporation Limited, Hangzhou 310000, China
}

\begin{abstract}
Large-scale coastal reclamation has become a significant land use issue worldwide for urban construction and economic development. The reclamation of coastal wetlands brings substantial economic benefits, however, the structure and function of coastal ecosystem are affected by drivers of human-caused landscape change. This research takes Hangzhou Bay and Zhoushan Islands as the case study to investigate the correlation between the coastal geomorphic complexity and the tidal range reduction rate, and to explore the control technical indexes of the design in reclamation area by a multidisciplinary approach that integrates the basic theories and quantitative methods of fractal geometry with the hydrodynamic mechanism of ocean dynamics. The results show that the coastal tidal range reduction rate is closely related to the coastline fractal dimension and patch shape index $(\mathrm{D}, \mathrm{S})$, and reveals the influence of the complexity of the coastal landscape on the tidal energy loss. In addition, based on model predictions, it can be found that the large-scale reclamation in Zhoushan will cause a serious decline in the complexity of the coastal landscape and the reduction of tidal energy, which is extremely detrimental to coastal disaster prevention. In the end, the scientific design theory and quantitative control indexes of reclamation are put forward to provide theoretical basis and design reference for future coastal reclamation and disaster prevention.
\end{abstract}

\section{Introduction}

Due to the growing demand for the resources, coastal reclamation has become a global land use practice. Since the middle of the 20th century, China has reclaimed a total of 13.4 million ha of tidal flats. ${ }^{[1]}$ Zhejiang is located in the southeast coastal area of China, with tortuous coasts, numerous islands and rich tidal flat resources. A total of 75134.3 ha of coastal wetland in Hangzhou bay were reclaimed in recent 4 decades.[2] Although the reclamation of coastal wetlands brings substantial economic benefits, it can lead to a series of adverse ecological impacts ${ }^{[2]}$ due to the lack of scientific theoretical guidance and effective quantitative control measures. The natural and complex coastal landscape has undergone permanent changes [3], and the service function of the ecosystem has also been severely weakened [4]. According to the 2017 National Marine Environmental Status Announcement, most of China's typical bays, estuaries, coastal wetlands, and other ecosystems are in a sub-healthy or unhealthy state. Among them, Hangzhou Bay is a heavily polluted sea area ${ }^{[5]}$. What's more serious is that the spatial shape of the estuary coast and the complicated boundary changes, resulting in an increase in tidal range, rising tide level ${ }^{[6]}$, and rising water level in the estuary ${ }^{[7]}$. This has increased the probability of flooding in coastal towns. For example, after the landfall of Typhoon Fitow, the 23th typhoon in2013 in China, the high tide level and rainstorms resulted in insufficient drainage capacity of the inland and outer rivers in many coastal cities in Zhejiang, the water level rose, and serious floods were caused. The direct economic loss reached 4.737 billion yuan ${ }^{[8]}$. It can be seen that these unscientific reclamation projects and the consequences have restricted the sustainable economic development of coastal areas in southeast China.

Zhoushan Archipelago is China's first new state-level area formed by archipelago and a key development area in the Hangzhou Bay Economic Belt. In the high-speed urban development, the reclamation of tidal flats in Zhoushan area mostly adopts 3 methods: harbor reclamation around a single island, reclamation connecting a small island and a big island, or connecting two adjacent small islands. This leads to a substantial change in the landscape structure along the coast, especially the natural and complex morphological characteristics of the coastline. Undoubtedly, it will have a more serious impact on the surrounding ecological environment. At present, there is still a lack of theoretical research on the influence of the complexity of coastal landforms on tidal energy dissipation, as well as its mechanism of tidal prevention and disaster reduction. Especially in the planning and design of reclamation area, there is a lack of quantitative control indexes and methods for the complexity of tidal flat landforms. Therefore, with Hangzhou Bay and Zhoushan Islands as a case study (Figure 1), the relationship between the

\footnotetext{
$\overline{{ }^{*} \text { Corresponding author: zhsx@zju.edu.cn }}$
} 
complexity of coastal geomorphology and tidal energy loss is studied, and quantifiable technical indicators of scientific reclamation are established, and ecological reclamation models that are conducive to coastal disaster prevention and mitigation are explored. It can provide a theoretical basis and design reference for the rational use of tidal flat wetland resources and the maintenance of the sustainable development of coastal areas.
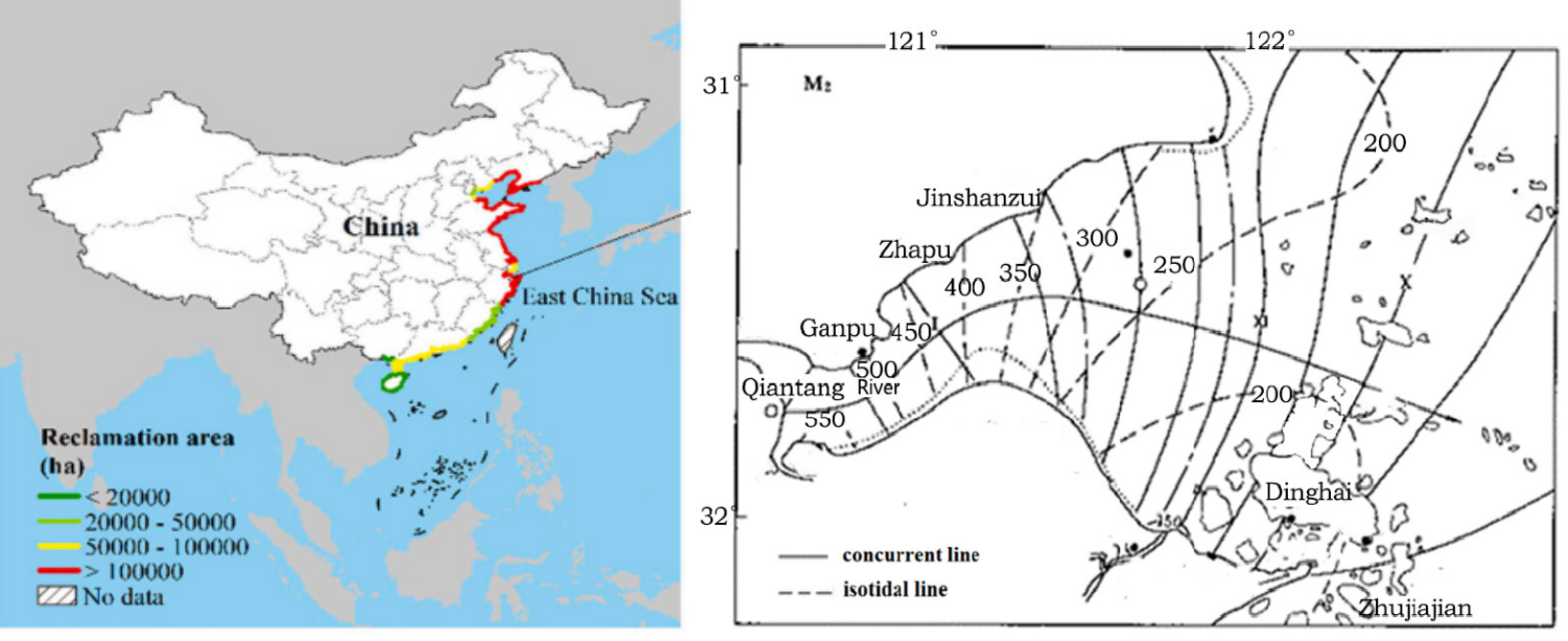

Fig. 1. Concurrent lines and isotidal lines of M2 tide peak in Hangzhou Bay

\section{Materials and Methods}

\subsection{Fractal Dimension and Calculation Method}

Fractal geometry theory takes irregular fragments as objects, such as the complex, chaotic, and rich forms of clouds, trees, coastlines in nature ${ }^{[9]}$, and its fragments are self-organized and self-similar. Fractal theory plays an important role in observing and analysing the relationship between various complex characteristic forms and their internal ecological functions in nature ${ }^{[10]}$. Fractal model is a research method based on this theory to explain the spatial shape and structure of objects. Fractal dimensions can quantitatively describe the complexity of irregular shapes, and reflect the naturalness and complexity of landscape composition and spatial pattern [11]. Fractal geometry and models have been successfully and widely used in quantitatively describing the fractal features of irregular objects such as coastlines and river networks [12-14]. A fractal model between the length of the observation object and the observation scale will be established in the calculation, and the grid method will be used ${ }^{[12-13]}$ to calculate the fractal dimension of the coastline. The calculation formula is as follows:

$$
N(\varepsilon) \propto \varepsilon^{-D}
$$

$$
\lg N(\varepsilon)=-D \lg \varepsilon+C
$$

Where $\mathrm{D}$ is the fractal dimension of the coastline, and it is determined by computing the slope of the plotted relationship. An irregular curve will have a fractal dimension higher than 1 but smaller than 2 . And $\mathrm{C}$ is a proportionality constant which allows for the relationship between $\mathrm{N}$ and $\varepsilon$ mentioned above. In this study, the number of boxes as a function of the grid size was calculated, and fractal dimensions were determined by Fractalize software. Normally, the more complex the shoreline shape is, the higher D is.

\subsection{Shape index of patch (S)}

The shape index (S) is an important indicator for quantitative analysis of landscape spatial pattern and landscape heterogeneity. It shows the regional ecological environment from a macro perspective ${ }^{[11]}$. The shape index of patch (S), assessing the complexity of coastal landscape patch by calculating the degree of deviation between the shape of a patch and the circle or square of the same area, was measured by the following equation $^{[15]}$.

$S= \begin{cases}\frac{P}{2 \sqrt{\pi A}}, & \text { In a round as reference } \\ \frac{0.25 P}{\sqrt{A}}, & \text { In a square as reference }\end{cases}$

where $\mathrm{P}$ is the perimeter of the patch, and $\mathrm{A}$ is the area of the patch. The value of shape index can reflect the size and the complexity of the landscape patch. The larger $\mathrm{S}$ is, the more complex the shape of patch is. Based on general the shape of study objects, this study measured the complexity of coastal islands and patches by using square objects of reference.

\section{Results \& Discussion}

\subsection{The Correlation between Coastal Geomorphic Complexity and Tidal Energy Loss}

According to statistics, from 1951 to 2005 , the total area of reclaimed tidal flats in Zhoushan has reached 199,200 $\mathrm{mu}$, which has fundamentally changed the landscape structure along the coast, especially the natural and 
complex morphological characteristics of the coastline. Therefore, taking Hangzhou Bay and the Zhoushan Islands as the main research objects (Figure 1), the impact of the landscape complexity of the tidal flow boundary (shoreline and islands) on the tidal energy loss is studied.

The tidal resonant along the coast of Zhejiang are formed by Pacific tidal waves and celestial tidal forces. Tides in Hangzhou Bay are dominated by semidiurnal M2 tide which is a progressive wave in East China Sea. Figure 1 shows the concurrent lines and isotidal lines of M2 tide peak in Hangzhou Bay. It can be seen from the concurrent lines that the progressive wave in the East China Sea first arrived at Zhujiajian in the Zhoushan Islands in the southern part of Hangzhou Bay. Due to the scattered distribution of Zhoushan Islands in the southern part of Hangzhou Bay, the propagation speed of tidal waves slowed down after being blocked by the islands. However, there are few islands in the northern part, and the tidal wave propagates fast. When it reaches the mouth of the bay, the concurrent lines turn close to north-south. The tidal range in Zhoushan Islands is much lower than other places.

In order to study the impact of the landscape complexity of the tidal path boundary (shoreline and islands) on the tidal current, Hangzhou Bay and Zhoushan Islands will be the main research objects. Taking Shupu at the mouth of the Qiantang River as a benchmark, the study area is divided into two areas in the north and south according to the central axis of Hangzhou Bay (Figure 2). Using fractal dimension model and shape index calculation method (formula 3), using fractalyse software, calculate the coastline fractal dimension $\mathrm{D}$ and patch shape index $\mathrm{S}$ of the two regions respectively. The calculation result is shown in Figure 3. It can be seen from Figure 3 that the $\mathrm{D}$ and $\mathrm{S}$ values in the south are larger than those in the north, and the shape index of the south is 2.14 times that of the north. The reason is that the southern part is the Zhoushan archipelago, which has islands of different shapes. The shape of the shoreline is much more complicated than that of the northern part. The landscape and landform are more complicated, and the resistance to tidal current is greater, and the tide energy is reduced more. It can be preliminarily shown that the complex geomorphic structure can reduce tidal energy and play a role in flood prevention and disaster reduction.

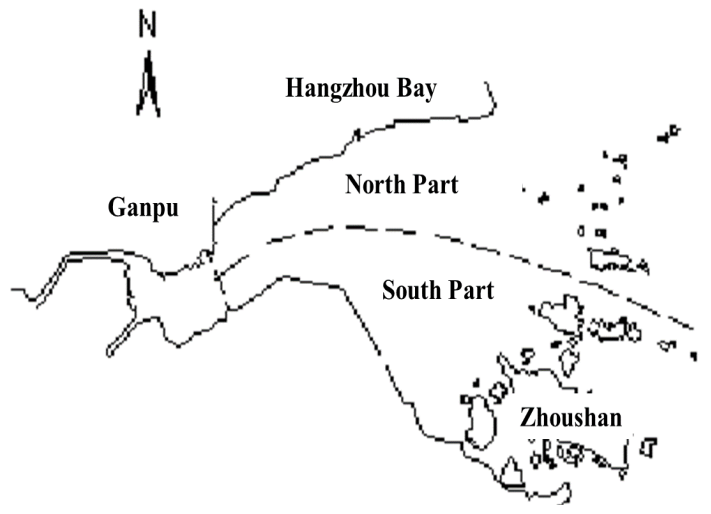

Fig. 2. Hangzhou Bay, Zhoushan Islands, and research boundaries

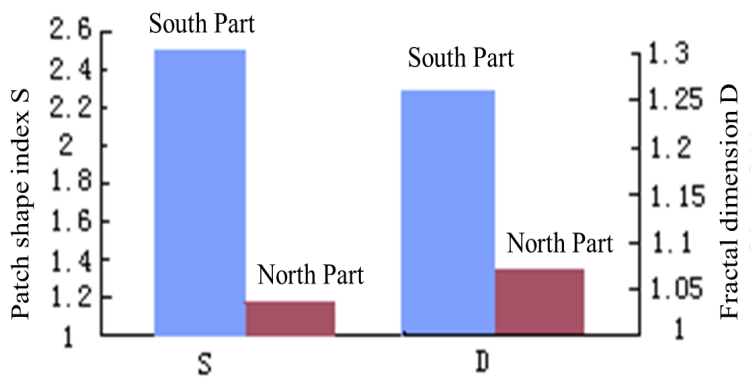

Fig. 3. Coastal Landscape Characteristic Indexes (S, D)

According to ocean dynamics theory, tidal energy is proportional to the square of tidal range, that is, for the same water surface area, the smaller the average tidal range, the smaller the tidal energy. So, it is reasonable to use the tidal range reduction rate, that is, the change in tidal range per unit distance, to indicate the degree of tidal energy loss. On the basis of my previous related research, the model was obtained (formula (4)) ${ }^{[16]}$.

$$
\Delta A=63.585 D+1.644 S-71.437
$$

The above formula shows that the tidal range reduction rate is closely related to the irregularity of the island and shoreline, the curvature of the boundary and the complexity of the spatial shape. The more tortuous the boundary of the coastline, the more complex the spatial shape, the larger the value of $\mathrm{D}$ and $\mathrm{S}$, the greater the tidal range drop per unit length, and the greater the resistance to tidal current. The tidal energy is proportional to the square of the tidal range, so the more tidal energy is lost. It can also be seen from this model that the $\mathrm{D}$ value of the coastline plays a decisive role in tidal energy dissipation. Therefore, it is recommended to use $\mathrm{D}$ and $\mathrm{S}$ values as the technical indicators to control the coastal complexity in the planning and design of reclamation. The model also provides a new method for evaluation and simulation of reclamation planning.

\subsection{Impact of Zhoushan Coastal Reclamation on Tidal Energy Loss and Countermeasures}

According to the "Zhoushan Tidal Flat Reclamation Planning Report", the current land reclamation plan for Zhoushan Island still uses the three traditional methods described in Chapter 1, which greatly reduces the tortuosity and complexity of the shoreline. Based on the 1:100,000 hardcopy topographic map of Zhoushan City in the early 1970 s and the digital topographic map of 2004 (after large-scale reclamation), this study used the model (formula 4) to compare and analyze the changes in the landscape index and tidal range reduction rate before and after the implement of reclamation plan. As shown in Table 1. 
Table 1. Changes in the landscape index and tidal range reduction rate before and after the implementation of the reclamation plan.

\begin{tabular}{|c|c|c|c|c|}
\hline & $\mathbf{D}$ & $\mathbf{S}$ & $\mathbf{N}$ & $\Delta A /(\mathbf{m m} / \mathbf{k m})$ \\
\hline Before & 1.144 & 3.66 & 98 & 7.32 \\
\hline After & 1.068 & 20.67 & 83 & 0.86 \\
\hline
\end{tabular}

It can be seen from Table 1 that due to traditional reclamation methods, the coastline shape of Zhoushan and nearby areas has undergone tremendous changes. The original tortuous shape of the shoreline became straight after reclamation. At the same time, the reduction of islands (the number of patches N) also reduces the complexity and richness of the spatial form, and reduces the length of the land and water boundary. Both the fractal dimension $\mathrm{D}$ and the shape index $\mathrm{S}$ are greatly reduced, which will greatly weaken the coastal hydrodynamic effect and reduce the tidal wave energy loss. The calculation results show that the tidal range reduction rate has been reduced by $88.3 \%$, which has adversely affected the disaster prevention work in the coastal and downstream estuaries and bay areas.

Therefore, determining the mode of coastal reclamation should start from determining the shape of the shoreline, and taking the fractal dimension as the main control index. It is necessary to follow the selfsimilarity of the natural coastline in Zhoushan area and try to imitate the natural morphological characteristics of the coastline. Meanwhile, on the basis of enriching the shape of the shoreline, referring to the auxiliary indicator of the shape index, the spatial layout of multiple islands should be retained. In this way, the fractal dimension and landscape shape index after the reclamation of the tidal flat are greater than or equal to the value before the reclamation to ensure that the complexity of the landscape after the project is not reduced. This can extend the length and space complexity of the land and water boundary, increase the resistance of tidal currents, reduce tidal energy and alleviate disasters.

\section{Conclusions}

This paper uses fractal dimension model, related calculation software (Fractalyse) and landscape quantitative indicators to calculate the coastline fractal dimension, shape index of island patch and tidal range reduction rate in typical areas, and study the correlation between each index and tidal range reduction rate. The results show that the tidal range reduction rate is closely related to the complexity of coastal morphology. The more tortuous the coastline and the more complex the spatial shape, the greater the value of $\mathrm{D}$ and $\mathrm{S}$, the greater the resistance to the tidal current, the greater the tidal energy loss.

In addition, the model was used to evaluate the reclamation plan in the Zhoushan area, and the results showed that the traditional reclamation method changed the complex topography of the shoreline. Both the fractal dimension $\mathrm{D}$ and the shape index $\mathrm{S}$ are greatly reduced, and the tidal range reduction rate is reduced by more than $88 \%$. It is extremely unfavorable for disaster prevention in coastal and estuaries and bay areas.

Therefore, when planning and designing tidal flat reclamation in a specific area, it is necessary to conduct detailed investigation on the landscape features that can withstand disasters, and protect the natural and complex spatial pattern as much as possible. It is recommended to adopt the quantitative indicators and methods of scientific reclamation based on "D $\backslash S$ " to improve and control the complexity of the landform, and to ensure that the complexity of the coastal landscape is not lower than the original value. The research results not only provide theoretical basis and practical guidance for the future large-scale tidal flat development of Hangzhou Bay and Zhoushan Islands, but also can enrich the basic theory and quantitative control indexes of tidal flat reclamation engineering design, which has very important scientific significance.

\section{Acknowledgements:}

This research is sponsored by the Scientific and Technical Project of Water Conservancy of Zhejiang Province (grant number: R14842, RC2008).

\section{References}

1. Fu, Y.B., Cao, K., Wang, F.(2010) Preliminary study of the methods used to evaluate the potential impacts of coastal reclamation.Ocean Development and Management., 27 (1):27-30.

2. Qiu,L.F.,Zhang,M.,Zhou,B.B.,Cui,Y.Z.,Yu,Z.L.,Liu ,T.,Wu,S.H.(2021)Economic and ecological tradesoffs of coastal reclamation in Hangzhou Bay, China. Ecological Indicators.,125:1:12.

3. Peng, B.R., Lin, C.C., Jin, D.(2013)Modeling the total allowable area for coastal reclamation: A case study of Xiamen, China.Ocean \& Coastal Management.,76:38-44.

4. Rao,H.H.,Lin,C.C.,Kong,H.(2014)Ecological damage compensation for coastal sea area uses. Ecological Indicators.,(38):149-158.

5. State Oceanic Administration (SOA).(2018)Announcement on the State of Marine Ecology and Environment of China in 2017.,www.soa.gov.cn/zwgk/hy

6. You,A.J.,Hang,Z.C.,He,R.Y.(2010)Characteristics and effecting factors of the tidal level in the Qiantangjiang River Estuary under changing environment. Journal of Marine Sciences., 28(1):1825.

7. Chen,X.W.,Liu,X.,Zhang,W.(2011)Shore reclamation in Pearl River Esturay and its impact analysis . Journal of Hohai University (Natural Sciences).,39(1):39-43. 
8. http://news.xinmin.cn/domestic/bjtj/2013/10/08/221 66339.html.

9. Wazawa,F., Kohiko,K., Kenaki,I.(2000)Landscape Environment Theory. Earth Society Press,Tokyo.

10. Griggs,J.A.(2018)Using fractal geometry to examine failed implants and prostheses. Dental Materials., 34(12): 1748-1755.

11. LI, B. L.(2000)Fractal geometry applications in description and analysis of patch patterns and patch dynamics. Ecological Modelling., 132(1/2): 33-50.

12. Zhu,X.H.,Cai,Y.L.(2004)Study on fractal dimension of Chinese Coastline and character.Advance in Marine Science.,22(2):156-162.

13. Zhang,S.X., Guo,Y.K.,Wang, Z.W. (2015)Correlation between flood frequency and geomorphologic complexity of rivers network-a case study of Hangzhou China. Journal of Hydrology.,527: 113-118.

14. Many,G., Durrieu de Madron,X.,Verney, R.,Bourrin, F.,Renosha,P.R.,Jourdin,F.,Gangloff,A.(2019)Geom etry, fractal dimension and settling velocity of flocs during flooding conditions in the Rhône ROFI. Estuarine, Coastal and Shelf Science., 219: 1-13.

15. Zhang S. X.,Wang, Z W.,Wu, S.N.(2020)Investigation of correlation between complexity of coastal landscape and water ecological environment.Marine Environment Science.,39:880-886.

16. Zhang S. X.,Wang, Z. W.,Wu, S.N.(2012) Landscape mechanism quantitative study on impact of tidal flat reclamation on the function of coastal disaster prevention: illustrated with Hangzhou Bay and Qiantang estuary. Journal of Zhejiang University(Engineering Science).,46:1281-1288. 